\title{
Robotic assisted cytoreductive surgery, removal of a recurrent disease in the right pericaval lymph node in a patient with ovarian cancer with the robotic Xi platform
}

\author{
Massoud Shoraka, MD', Hadeer Metwally, MD', Semiramis Carbajal-Mamani, MD², \\ Joel Cardenas-Goicoechea, MD, MBA ${ }^{1}$ \\ Departments of ${ }^{1}$ Obstetrics and Gynecology, ${ }^{2}$ Medicine, University of Florida College of Medicine, Gainesville, FL, USA
}

\section{Objective}

The standard approach for recurrent ovarian cancer is laparotomy. In this video, we present a cytoreductive surgery using the robotic Xi platform to remove a $2.7 \mathrm{~cm}$ pericaval tumor.

\section{Methods}

A narrative video demonstration of robotic-assisted surgery to remove recurrent ovarian cancer in a pericaval lymph node. A 62-year-old female presented with recurrent carcinoma of the pericaval lymph node. After 40 months of surveillance, the patient was asymptomatic, but a computed tomography $(\mathrm{CT})$ scan showed an isolated mass $(2.7 \times 2.3 \mathrm{~cm})$ in the right pericaval lymph node. Her cancer antigen (CA)-125 level increased from 26 to $46 \mathrm{U} / \mathrm{mL}$. The robotic Xi platform was used to remove the metastatic lymph nodes. The first step was diagnostic laparoscopy. The second step was robotic port placement below the umbilicus. The third step was dissection and identification of landmarks, and the last step was removal of the tumor and closure.

Results

The metastatic lymph nodes were removed. The patient was discharged on postoperative day 1 and had no postoperative complications. Her CA-125 level dropped to $17 \mathrm{U} / \mathrm{mL}$ two weeks after surgery. Pathology showed metastatic high-grade serous carcinoma in one lymph node, consistent with the patient's known primary. Two additional lymph nodes were removed and negative for carcinoma. Pelvic washings were negative for malignancy.

\section{Conclusion}

Robotic-assisted surgery is safe and feasible in selected patients with isolated recurrent disease.

Keywords: Ovarian neoplasm; Recurrence; Lymph nodes; Cytoreduction surgical procedures; Robotic surgical procedures

The management of recurrent ovarian carcinoma is complex and depends on the time of recurrence after surgery and tumor burden. Treatment options include chemotherapy and radical cytoreductive surgery. Surgery is usually indicated in patients with good physical performance and a long interval from primary treatment. Recently, two clinical trials have suggested that secondary surgery extends survival in this setting. The results of the SOC1/SGOG-OV2 and AGO DESKTOP III/ ENGOT-ov20 clinical trials were presented at the American Society of Clinical Oncology 2020 annual meeting $[1,2]$. The gold standard approach is laparotomy for the resection of all diseases (RO). Such a complex operation may require a large
Received: 2021.06.24. Revised: 2021.07.21. Accepted: 2021.07.26. Corresponding author: Joel Cardenas-Goicoechea, MD, MBA Department of Obstetrics and Gynecology, University of Florida College of Medicine, 1600 SW Archer Rd, Gainesville 32610, FL, USA

E-mail: joelcardenas@yahoo.com

https://orcid.org/0000-0002-0589-5785

Articles published in Obstet Gynecol Sci are open-access, distributed under the terms of the Creative Commons Attribution Non-Commercial License (http://creativecommons. org/licenses/by-nc/3.0/) which permits unrestricted non-commercial use, distribution, and reproduction in any medium, provided the original work is properly cited.

Copyright $\odot 2021$ Korean Society of Obstetrics and Gynecology 


\section{Obstetrics \& Gynecology Science}

Massoud Shoraka, et al. Robotic removal of pericaval lymph node

incision and is associated with an increased risk of adverse events such as bleeding, blood transfusion, incisional hernia, infection, and prolonged hospital stay. Recently, roboticassisted surgery has been described by single- and multiinstitution experiences.

The first three generations of the robotic da $\mathrm{Vinc}^{\circledR}$ platform (da Vinci ${ }^{\circledR}$, da Vinci $S^{\circledast}$, and da Vinci Si ${ }^{\circledR}$ [da Vinci ${ }^{\circledR}$ Intuitive Surgical Inc., Sunnyvale, CA, USA]) had limitations in performing abdominal and pelvic surgery because it required additional maneuvers. In 2014, the da Vinci $X^{\circledR}{ }^{\circledR}$ platform (Intuitive Surgical, Inc. Sunnyvale, CA, USA) was introduced to the global market, and our practice implemented the platform in 2016. Our objective was to describe a robotic approach using the Xi platform.

A 62-year-old female with a history of stage IIC high-grade serous carcinoma of the right ovary underwent robotic-assisted radical cytoreductive surgery on December 7, 2017. Surgery included diagnostic laparoscopy, complete cytoreductive surgery (R0), radical hysterectomy, right parametrectomy, bilateral salpingo-oophorectomy, bilateral pelvic lymph node dissection, para-aortic lymph node dissection, and infracolic omentectomy. Her cancer antigen (CA)-125 level at the time of diagnosis was $51 \mathrm{U} / \mathrm{mL}$. After surgery, the patient received six cycles of adjuvant chemotherapy with carboplatin plus paclitaxel. Genetic testing revealed a RAD51D mutation.

During surveillance, the patient's CA-125 level ranged from 8-26 U/mL, but on April 13, 2021, it rose to $46 \mathrm{U} / \mathrm{mL}$. A computed tomography scan showed a new mass in the right pericaval lymph node measuring $2.7 \times 2.3 \mathrm{~cm}$, which raised suspicions of recurrent disease. There were no other areas of recurrent disease (Fig. 1). The patient was asymptomatic, with a body mass index (BMI) of $24.53 \mathrm{~kg} / \mathrm{m}^{2}$. Her medical history included gastroesophageal reflux disease, high cholesterol levels, and osteoarthritis. Her surgical history included prior cytoreductive surgery and left hip arthroplasty. She had a family history of breast cancer, high cholesterol, diabetes, coronary artery disease, and uterine and colon cancer. This case was presented to our multidisciplinary tumor board. The options discussed included chemotherapy and surgery.

Step 1: Diagnostic laparoscopy was performed. A 5-mm port was placed in the upper left quadrant. A systematic review of the abdomen and pelvis revealed no gross disease.

Step 2: Four robotic trocars, $8 \mathrm{~mm}$ in size, were placed as the following locations. The robotic camera trocar was placed in the midline and $1 \mathrm{~cm}$ below the umbilicus. Two trocars were placed 6 and $12 \mathrm{~cm}$ from the midline trocar. A fourth robotic trocar was placed in the left flank, $10 \mathrm{~cm}$ from the midline trocar. All robotic trocars were at the same level (Fig. 2). The Xi robot was docked in a standard fashion (left lateral docking).

Step 3: The peritoneum was incised and dissected cephalad
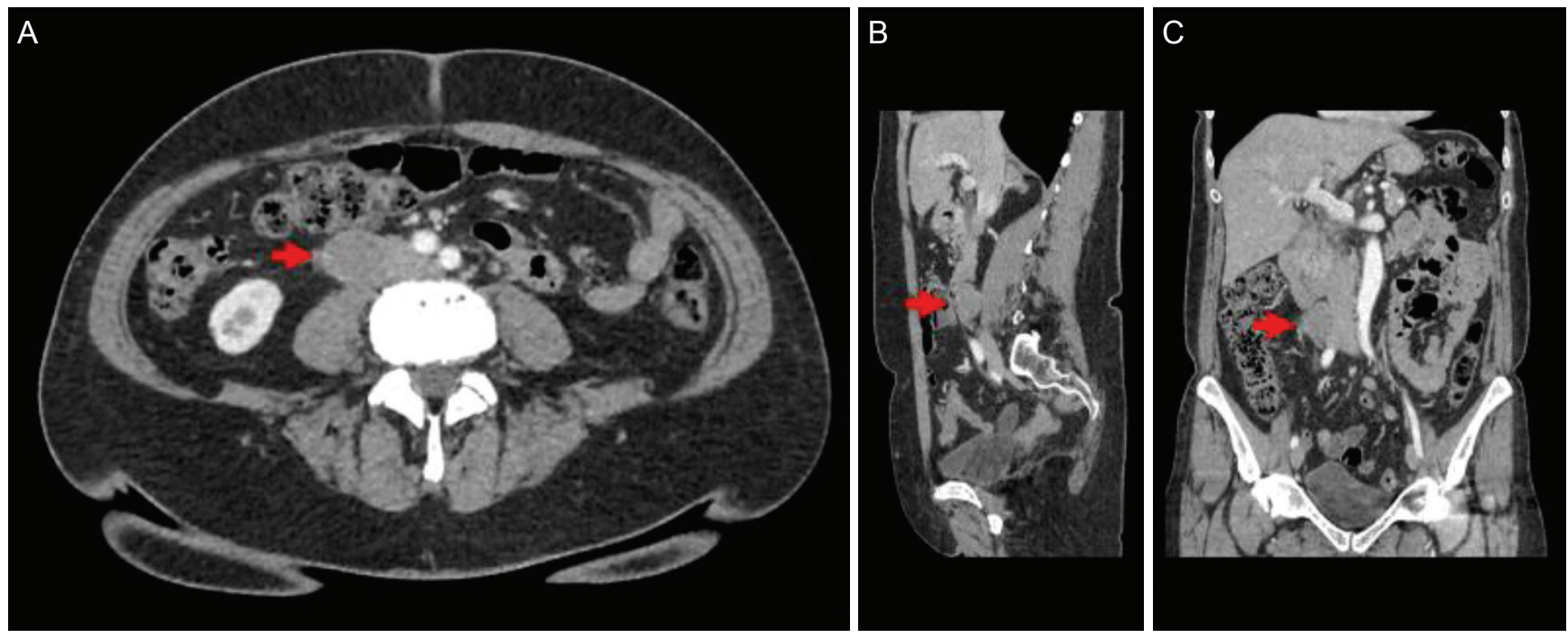

Fig. 1. Abdominal and pelvic computed tomography scan abdomen and pelvis showing pericaval lymph node (arrows). (A) Axial view. (B) Lateral view. (C) Coronal view. 


\section{Obstetrics \& Gynecology Science}

Vol. 64, No. 6, 2021

along the aorta. The landmarks for the dissection were the following: superiorly, peritoneal reflection of the duodenum and Gerota fascia; inferiorly, the right common iliac artery; laterally, the right ureter; and medially, the aorta. The bulky tumor was sequentially dissected with a combination of blunt and sharp dissection with monopolar and bipolar cautery. The lymphatic channels and fellow veins were isolated and sealed with cautery. The specimen was removed intact in one piece, placed and secured in a 10-mm laparoscopic endobag, and removed through the infraumbilical port. Two additional para-aortic lymph nodes and cysts were removed. At the end of the procedure, no gross residual disease (RO) was observed. A fibrin sealant (10 mL) was placed along the inferior vena cava.

Step 4: The peritoneum was reapproximated using three robotic clips (Fig. 2). The estimated blood loss was $20 \mathrm{~mL}$, and the operative time was 146 minutes.

The patient was admitted for observation and discharged home on postoperative day 1 . Pathological assessment identified a specimen of $5.1 \times 3.1 \times 2.3 \mathrm{~cm}$ in size, with metastatic high-grade serous carcinoma consistent with the patient's known primary.

The risk of recurrent ovarian cancer is high, and the management is complex. Recently, three clinical trials have been presented to evaluate the role of surgery. The Desktop and SOC-1 trials suggest that cytoreductive surgery plays a role in improving survival. The GOG 213 clinical trial did not find a survival advantage of surgery when compared to chemotherapy [3]. Laparoscopy and robotic-assisted surgery have been introduced in many institutions as an alternative to open surgery for secondary cytoreductive surgery, but published data are limited [4-8]. The National Comprehensive Cancer Network guidelines suggest that minimally invasive techniques
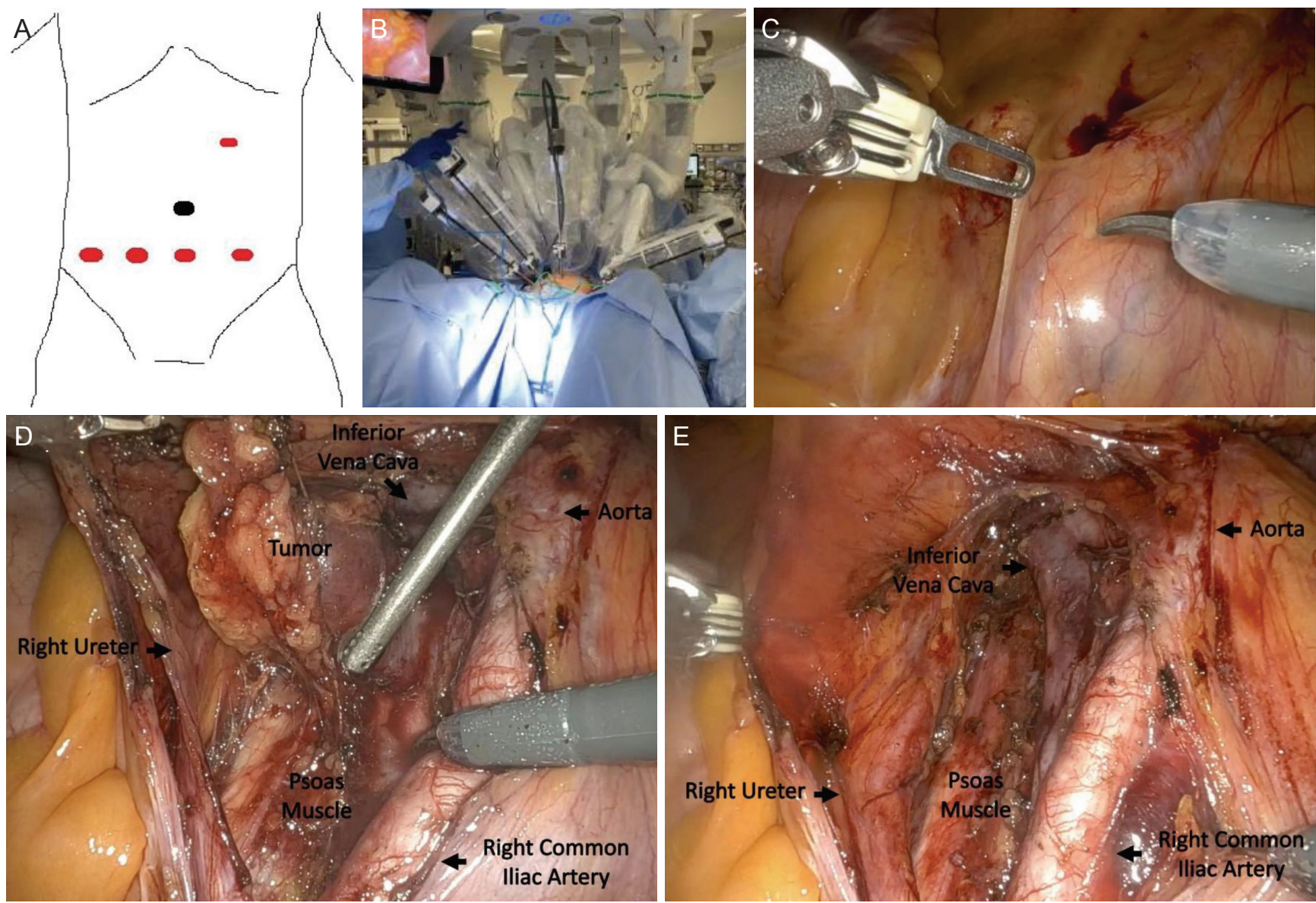

Fig. 2. Surgical procedure. (A) Port placement. Accessory port at left upper quadrant. (B) Left lateral side docking with an abdominal approach. (C) Cephalad incision of peritoneum along the aorta. (D) Identification of landmarks, dissection of tumor, and isolation and sealing of the lymphatic channels and fellow veins. (E) End of procedure. 


\section{Obstetrics \& Gynecology Science}

Massoud Shoraka, et al. Robotic removal of pericaval lymph node

can be used for selected patients for interval debulking procedures. Patients who are unwilling to be optimally debulked using minimally invasive techniques should be converted to open procedures [9].

The advantages of the da Vinci $X^{\circledR}{ }^{\circledR}$ (Intuitive Surgical Inc.) are its versatility for multi-quadrant surgery with very simple maneuvers, thinner arms, increased range of motion, and same-size trocars that enable camera placement in any port. The Xi platform allows the surgeon to perform pelvic surgery and then move to upper abdominal surgery by rotating the pivot boom without changing the patient cart location.

With simple and reproducible steps, our video showed the feasibility and safety of robot-assisted surgery with the benefits of low blood loss, low levels of surgical pain, and short hospital stays. Features of the ideal candidate for consideration for robotic-assisted surgery for recurrent ovarian cancer may include the presence of an isolated tumor, absence of ascites and carcinomatosis, adequate exposure, and access to the tumor. A standard open approach should be reserved for patients with extensive adhesions and multiple sites of recurrence. Since there is no prospective or randomized trial that compared open surgery to robotic-assisted surgery, a tumor registry or more publications are needed to confirm the safety of the procedure. A longer follow-up period of survival is also warranted. Robotic-assisted surgery is safe and feasible for selected patients with isolated recurrent ovarian cancer or for laparotomy in selected patients.

\section{Conflict of interest}

No potential conflict of interest relevant to this article was reported.

\section{Ethical approval}

This study did not require approval from the Institutional Review Board because no patient data were included in this study.

\section{Patient consent}

Written informed consent was obtained from the patient for the publication of this case report and accompanying images.

\section{Funding information}

No financial support was used for this study.

\section{Acknowledgments}

This video has not been published elsewhere, and it is not accepted for publication or under review for publication in any other journal.

\section{Video clip}

Video can be found with this article online at https://doi. org/10.5468/ogs.21202.

\section{References}

1. AGO Studiengruppe Harter P, Sehouli J, Meier W, Reuss A, Hillemanns $P$, Hasenburg $A$, et al. Randomized phase III study to evaluate the impact of secondary cytoreductive surgery in recurrent ovarian cancer-final analysis of AGO DESKTOP III/ENGOT- ov20. Geburtshilfe Frauenheilkd 2020;80:e79.

2. Zang R, Zhu J, Shi T, Liu J, Tu D, Yin S, et al. A randomized phase III trial of secondary cytoreductive surgery in later recurrent ovarian cancer: SOC1/SGOG-OV2. J Clin Oncol 2020 May 20 [Epub]. https://ascopubs.org/ doi/10.1200/JCO.2020.38.15_suppl.6001.

3. Coleman RL, Spirtos NM, Enserro D, Herzog TJ, Sabbatini $P$, Armstrong DK, et al. Secondary surgical cytoreduction for recurrent ovarian cancer. $N$ Engl J Med 2019;381:1929-39.

4. Feuer GA, Lakhi N, Barker J, Salmieri S, Burrell M. Perioperative and clinical outcomes in the management of epithelial ovarian cancer using a robotic or abdominal approach. Gynecol Oncol 2013;131:520-4.

5. Holloway RW, Brudie LA, Rakowski JA, Ahmad S. Robotic-assisted resection of liver and diaphragm recurrent ovarian carcinoma: description of technique. Gynecol Oncol 2011;120:419-22.

6. Escobar PF, Levinson KL, Magrina J, Martino MA, Barakat RR, Fader AN, et al. Feasibility and perioperative out- 


\section{Obstetrics \& Gynecology Science}

Vol. 64, No. 6, 2021

comes of robotic-assisted surgery in the management of recurrent ovarian cancer: a multi-institutional study. Gynecol Oncol 2014;134:253-6.

7. Magrina JF, Cetta RL, Chang YH, Guevara G, Magtibay PM. Analysis of secondary cytoreduction for recurrent ovarian cancer by robotics, laparoscopy and laparotomy. Gynecol Oncol 2013;129:336-40.

8. Nezhat FR, Finger TN, Vetere P, Radjabi AR, Vega $M$, Averbuch $L$, et al. Comparison of perioperative outcomes and complication rates between conventional versus robotic-assisted laparoscopy in the evaluation and management of early, advanced, and recurrent stage ovarian, fallopian tube, and primary peritoneal cancer. Int J Gynecol Cancer 2014;24:600-7.

9. National Comprehensive Cancer Network. NCCN clinical practice guide-lines: ovarian cancer. Version: 1.2021 [Internet]. Plymouth Meeting (PA): National Comprehensive Cancer Network; c2021 [cited 2021 Jun 24]. Available from: https://www.nccn.org/guidelines/guidelinesdetail? category=1\&id=1453. 